\title{
The relevance of center vortices *
}

\author{
C. Alexandrou ${ }^{\mathrm{a}}$, M. D'Elia ${ }^{\mathrm{b}}$ and Ph. de Forcrand ${ }^{\mathrm{c}}$ \\ ${ }^{\text {a} U n i v e r s i t y ~ o f ~ C y p r u s, ~ C Y-1678 ~ N i c o s i a, ~ C y p r u s ~ a n d ~ P S I, ~ C H-5232 ~ V i l l i g e n, ~ S w i t z e r l a n d ~}$ \\ ${ }^{\mathrm{b}}$ University of Pisa and INFN, I-56127 Pisa, Italy \\ ${ }^{\mathrm{c} E T H, ~ C H-8092 ~ Z u ̈ r i c h, ~ S w i t z e r l a n d ~}$
}

We show remnants of chiral symmetry breaking in the center-projected theory. We construct and study an unambiguous definition of center vortices.

\section{Non-perturbative effects in the center- projected theory}

The standard approach to identify center vortices proceeds through gauge fixing. In [1, we fixed an $S U(2)$ ensemble to Direct Maximal Center (DMC) gauge, by iteratively maximizing

$Q\left(\left\{U_{\mu}\right\}\right) \equiv \sum_{x, \mu}\left(\operatorname{Tr} U_{\mu}(x)\right)^{2}$.

After factorizing gauge-fixed links as $U_{\mu}(x)=$ $\operatorname{sign}\left(\operatorname{Tr} U_{\mu}(x)\right) \times U_{\mu}^{\prime}(x)$ we studied the properties of the ensemble $\left\{U_{\mu}^{\prime}(x)\right\}$, which by construction contains no center vortices. We showed that all non-perturbative features had disappeared: confinement, chiral symmetry breaking, and nontrivial topology. We have now looked at the center-projected theory $\left\{\operatorname{sign}\left(\operatorname{Tr} \mathrm{U}_{\mu}(\mathrm{x})\right)\right\}$, to see if it inherits the non-perturbative properties of the original. As in [2], we observe that the string tension is consistent with its $S U(2)$ value. We measure the quark condensate $\langle\bar{\psi} \psi\rangle\left(m_{q}\right)$ as a probe of chiral symmetry breaking. Fig. 1 shows that it clearly extrapolates linearly to a non-zero value as $m_{q} \rightarrow 0$. Furthermore, it diverges as $1 / m_{q}$ for very small quark masses, revealing the presence of a few extremely small eigenvalues which may be caused by the non-trivial topological content of the $S U(2)$ configuration. Note the similarity of Fig.1 with the quenched condensate observed with domain-wall fermions [3]. However, the associated quasi-zero modes appear to be strongly localized and not chiral (i.e., $\bar{\psi} \gamma_{5} \psi \sim 0$ ).

${ }^{*}$ Talk presented by $\mathrm{Ph}$. de Forcrand

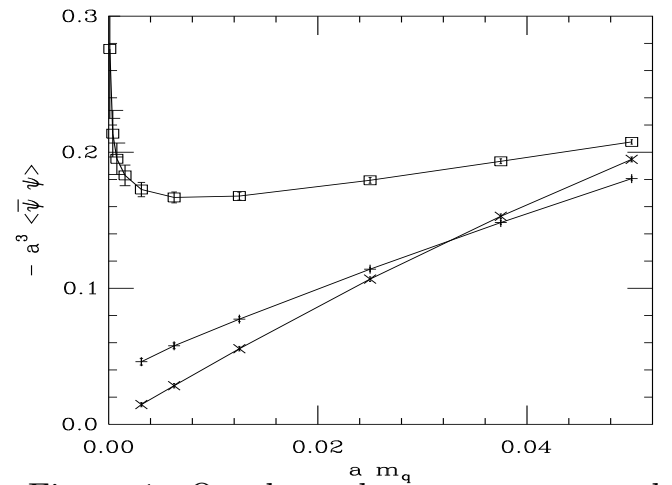

Figure 1. Quark condensate versus quark mass, in the original $(+)$, the center-vortex free $(\mathrm{x})$, and the center-projected (squares) theories.

\section{Unambiguous center-vortex cores}

The local implementation of the DMC gaugefixing Eq.(1) is ambiguous, leading to many local maxima. The properties of $P$-vortices obtained from different Gribov copies can be dramatically different 画. Here we define an unambiguous gauge condition. Note first that DMC is equivalent to maximizing $\sum_{x, \mu} \operatorname{Tr}_{\text {adj }} U_{\mu}(x)$ since $\operatorname{Tr}_{\text {adj }} U=2(\operatorname{Tr} U)^{2}-1$. The idea is thus to smooth the center-blind, adjoint component of the gauge field as much as possible, then to read the center component off the fundamental gauge field. Therefore, Maximal Center Gauge is just another name for adjoint Landau gauge.

The problem of Gribov copies in the fundamental Landau gauge was solved in [5] by computing the covariant Laplacian $\Delta_{x y}=2 d \delta_{x y}-$ $\sum_{ \pm \hat{\mu}} U_{ \pm \hat{\mu}}(x) \delta_{x \pm \hat{\mu}, y}$ and its lowest-lying eigenvec- 
tor $\vec{v}$. At each site, $v(x)$ has 2 complex color components. The Laplacian gauge condition consists of rotating $v(x)^{\dagger}$ along direction $(1,1)$ at all sites. We follow this construction for the adjoint representation. The covariant Laplacian is now constructed from adjoint links $U^{a b}=$ $\frac{1}{2} \operatorname{Tr}\left[U \sigma^{a} U^{\dagger} \sigma^{b}\right], a, b=1,2,3$. It is a real symmetric matrix. The lowest-lying eigenvector $\vec{v}$ has 3 real components $v_{i}, i=1,2,3$ at each site $x$. One can apply a local gauge transformation $g(x)$ to rotate it along some fixed direction, e.g., $\sigma_{3}$. Note, however, that this does not specify the gauge completely: Abelian rotations around this reference direction are still possible. Here they are of the form $e^{i \theta(x) \sigma_{3}}$. What we have achieved at this stage is a variation of Maximal Abelian Gauge which is free of Gribov ambiguities. This Laplacian Abelian Gauge has been proposed in [6], which also shows that monopoles can not only be identified through the DeGrand-Toussaint procedure in the Abelian projected theory, but should be directly identifiable by the condition $|v(x)|=0$ for smooth fields. Abelian monopole worldlines appear naturally as the locus of ambiguities in the gauge-fixing procedure: the rotation to apply to $v(x)$ cannot be specified when $|v(x)|=0$.

To fix to center gauge, we must go beyond Laplacian Abelian Gauge and specify the Abelian rotation $e^{i \theta(x) \sigma_{3}}$. This is done most naturally by considering the second-lowest eigenvector $\overrightarrow{v^{\prime}}$ of the adjoint covariant Laplacian, and requiring that the plane $\left(v(x), v^{\prime}(x)\right)$ be parallel to, say, $\left(\sigma_{3}, \sigma_{1}\right)$ at every site $x$. This fixes the gauge completely, except where $v(x)$ and $v^{\prime}(x)$ are collinear. Collinearity occurs when $\frac{v_{1}}{v_{1}^{\prime}}=\frac{v_{2}}{v_{2}^{\prime}}=\frac{v_{3}}{v_{3}^{\prime}}$, i.e. 2 constraints must be satisfied. Thus, gauge-fixing ambiguities have codimension 2: in $4 d$, they are $2 d$ surfaces. They can be considered as the centervortex cores for the following reasons:

First, note the analogy with fluid dynamics: in that context, a vortex refers to a helical flow, with the vortex core at the center of the helix. At the core, the centripetal acceleration vanishes, so that velocity and acceleration are collinear. Indeed, this collinearity condition has been used to identify vortex cores in $3 d$ fluid flow [7].

Consider now the intersection of our $2 d$ center- vortex core with some plane $(\mu, \nu)$ at a point $x_{0}$. As one describes a small loop around $x_{0}$ in the plane $(\mu, \nu)$, the rotation $\theta(x)$ necessary to maintain $\left(v(x), v^{\prime}(x)\right)$ parallel to $\left(\sigma_{3}, \sigma_{1}\right)$ varies by $2 \pi$, reflecting the gauge singularity at $x_{0}$. But this is a rotation of the adjoint field, so that the gauge rotation of the fundamental field as one goes around the small loop will be $e^{-i \frac{1}{2} 2 \pi \sigma_{3}}=-\mathbf{1}$. A small Wilson loop around $x_{0}$ will have trace -1 in the fundamental representation. This shows that center-vortex cores are aptly named, since they are indeed dual to -1 small Wilson loops. If the gauge field is smooth, these -1 loops will also be identified by the usual procedure, consisting of extracting $\operatorname{sign}\left(\operatorname{Tr} \mathrm{U}_{\mu}(\mathrm{x})\right)$ and computing the $Z(2)$ plaquette. The so-called $P$-vortices constructed that way are indeed almost dual to the center-vortex cores, but not exactly. This is because they are obtained by a somewhat arbitrary, non-linear recipe. In our construction, unlike in $\mathrm{DMC}$, the center-vortex cores where gauge fixing is ambiguous are the fundamental objects.

Our Laplacian Center Gauge solves the Gribov problem. It does not require an underlying lattice, but can be studied in the continuum like the original Laplacian gauge [8]. And it exhibits the center-vortex cores as an intrinsic property of the gauge field, independently of the gauge condition chosen: one could specify to rotate $v(x)$ and $v^{\prime}(x)$ at every site along arbitrary, $x$-dependent directions rather than $\sigma_{3}$ and $\sigma_{1}$. The center-vortex cores will be unchanged.

Still, some arbitrariness remains in two respects: (i) Another discretization of the covariant Laplacian could be used, with higher-derivative, irrelevant terms. This will affect the location and density of the center-vortex cores.

(ii) Another choice of covariant eigenvectors $\vec{v}, \overrightarrow{v^{\prime}}$ could be made. While it seems natural to choose for $\vec{v}$ the lowest-lying eigenmode to maximize the smoothness of the gauge, the choice of $\overrightarrow{v^{\prime}}$ appears less crucial. The third eigenvector of the Laplacian could be taken as well, or even the second eigenvector of a Laplacian modified as per (i). Under a different choice of $\overrightarrow{v^{\prime}}$, the centervortex cores will change, but not the monopoles, defined by $|v(x)|=0$. The $1 d$ monopole worldlines remain embedded in the $2 d$ center-vortex 


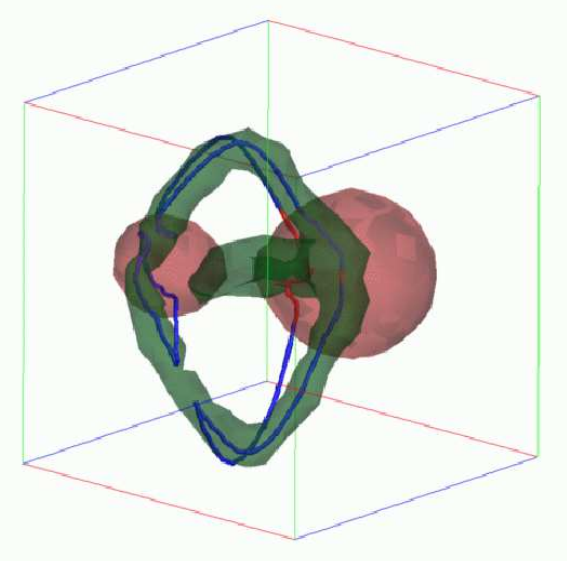

Figure 2. Time-slice of a cooled two-instanton (pink) configuration. The blue loops are centervortex cores. The green surface indicates high $Z(2)$ action density.

cores. In fact, one may view the center-vortex cores as the sheet spanned by the Dirac string of the monopoles in the adjoint representation.

We have applied Laplacian Center Gauge fixing and center projection to an ensemble of $S U(2)$ configurations. As in [1], the string tension, the quark condensate and the topological charge all vanish upon removal of the $P$-vortices. The $Z(2)$ string tension is consistent with its $S U(2)$ value, and the $Z(2)$ quark condensate behaves as in Fig.1. The main difference is that the $P$-vortex density is higher than with DMC ( 11\% vs $\sim 5.5 \%)$. The same effect was observed for the monopole density in Laplacian Abelian Gauge [6].

We have also applied our procedure to classical configurations. Fig.2 shows a cooled twoinstanton configuration. Note the double loop of vortex cores, which shows interesting signs of self-intersection, as required in the continuum [9]. The green area shows the $Z(2)$ action density, coming from $P$-vortices: while overall agreement is quite good between vortex cores and $P$-vortices, the latter are more sensitive to UV fluctuations and show some spurious structure. Fig. 3 shows a caloron, i.e. a large instanton at finite temperature. As explained in [10], it decomposes into a pair of monopoles, identifiable by their action and topological charge densities. The center-vortex cores recognize these monopoles: they pierce them and $|v|$ vanishes precisely at their center, as shown by the change of color $\left(\mathrm{red}=v, v^{\prime}\right.$ par-

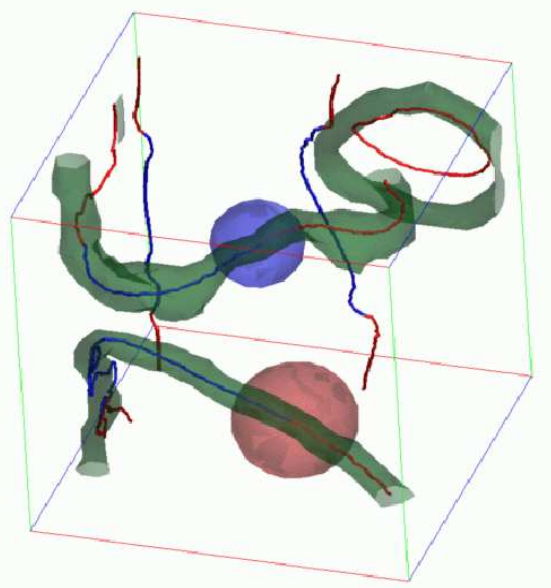

Figure 3. Time-slice of a cooled caloron configuration. The two monopoles are pink and blue. The center-vortex cores (closed loops) pierce the monopoles at their center (where $|v|=0$ ). The green surface indicates high $Z(2)$ action density.

allel; blue=anti-parallel). Again the $P$-vortices give a slightly modified picture.

Finally, our procedure readily generalizes to $S U(N)$ : complete gauge-fixing is achieved by rotating the first $\left(N^{2}-2\right)$ eigenvectors of the adjoint Laplacian along some reference directions. Ambiguities arise whenever these $\left(N^{2}-2\right)$ eigenvectors [each with $\left(N^{2}-1\right)$ real components] become linearly dependent. This again defines codimension2 center-vortex cores.

\section{REFERENCES}

1. Ph. de Forcrand and M. D'Elia, Phys. Rev. Lett. 82 (1999) 4582.

2. L. Del Debbio et al., Phys. Rev. D 55 (1997) 2298.

3. E.g. P. Chen et al., hep-lat/9811013, Fig.2.

4. T.G. Kovacs and E.T. Tomboulis, heplat/9905029.

5. J.C. Vink and U.-J. Wiese, Phys. Lett. B 289 (1992) 122.

6. A.J. van der Sijs, hep-lat/9803001; heplat/9809126.

7. D. Kenwright and R. Haimes, Proceedings of IEEE visualization (Oct. 1997) 413.

8. P. van Baal, hep-lat/9411047.

9. M. Engelhardt and H. Reinhardt, hepth/9907139.

10. M. Garcia Pérez et al., hep-lat/9903022. 